\title{
Vorinostat: a histone deacetylases (HDAC) inhibitor ameliorates traumatic brain injury by inducing iNOS/Nrf2/ARE pathway
}

\author{
Jinyu Xu, Jun Shi, Jiaming Zhang, Yun Zhang
}

Department of Emergency, Wuxi People's Hospital, Wuxi, China 214000

\begin{abstract}
The present investigation evaluates the protective effect of vorinostat on neuronal cells in the traumatic brain injury (TBI) and also postulates the possible mechanism of its action. Marmarou's weight-drop model was used to induce the TBI. Further, animals were treated with vorinostat $100 \mathrm{mg} / \mathrm{kg}$ intraperitoneally $30 \mathrm{~min}$ before the TBI induction. Neurological score and brain water content were determined in all the groups and thereafter oxidative stress parameters and adenosine triphosphate (ATP) content were determined in the neuronal tissues of TBI mice. Western blot assay and reverse transcription polymerase chain reaction (RT-PCR) was performed for the determination of the expression of several proteins in the neuronal tissues. Moreover, immunohistochemical staining and terminal deoxynucleotidyl transferase dUTP nick end labeling (TUNEL) assay was also done on the neuronal tissues of TBI mice. Data of the study reveal that treatment with vorinostat significantly reduces the altered level of grip test scores and water content in the brain of traumatic injured mice. Moreover, the altered level of oxidative stress parameters, translocation of nuclear factor erythroid 2-related factor 2 (Nrf2) and ATP content was attenuated by treating TBI mice with vorinostat. Also treatment with vorinostat ameliorates the altered expression of $p-A k t, N F-\kappa B$, iNOS and caspase by the western blot assay in the neuronal tissue of TBI mice and mRNA level of HO-1 and NQO-1 significantly enhanced in vorinostat compared to the negative control group. Furthermore, the TUNEL assay also reveals that the apoptosis of neuronal cells was significantly $(p<0.01)$ reduced in the vorinostat-treated group compared to the negative control group. The present study concludes that vorinostat protects the neuronal injury in TBI mice by reducing the altered level of oxidative stress and inflammatory response.
\end{abstract}

Key words: vorinostat, traumatic brain injury, apoptosis, oxidative stress and inflammation.

\section{Introduction}

In the modern society, the traumatic brain injury (TBI) is a major health problem that causes disability and death in the young aged population [13]. In $\mathrm{TBI}$, the secondary injury occurs due to inflammation, oxidative stress, increased vascular permeability, excitotoxicity and neuronal apoptosis [16]. The reported study reveals that neuronal damage in TBI is further stimulated by secondary injuries [7]. Although several efforts were made for the management of secondary injury-induced neuronal damage, clinical significance has not been observed. Thus it is important to develop new therapeutic strategies. Oxidative stress plays a vital role in the development of the secondary injury [11]. In neuronal cells, production of reactive oxygen

\section{Communicating authors}

Jinyu Xu and Yun Zhang, Department of Emergency, Wuxi People's Hospital, 299\# Qingyang Road, Wuxi, China 214000,

phone: 0086-051085350685, e-mail: JoeMozellevo@yahoo.com (J.X.), yppqp4e@163.com 
species (ROS) enhanced that leads to neuronal cell death by DNA damage [4]. Nrf2 protein reduces oxidative stress. Nrf2/ARE signaling pathway reported for its neuroprotective effect on the basis of anti-oxidant and anti-inflammatory activity [6].

Vorinostat is a histone deacetylases (HDAC) inhibitor and chemically a suberanilohydroxamic acid [12]. HDAC inhibitors are reported for their anti-depressant, anti-epileptic properties and are recommended for neurodegenerative diseases [1]. Moreover HDAC inhibitor has strong anti-inflammatory, anti-cancer, anti-oxidant and anti-HIV properties [8]. Vorinostat has been approved for the management of cutaneous T cell lymphoma clinically by the US FDA [9]. Vorinostat has also shown activity against cystic fibrosis and $\alpha_{1}$-antitrypsin deficiency [10]. Thus, the present investigation evaluates the protective effect of vorinostat against TBI-induced neuronal injury.

\section{Material and methods \\ Animal}

Male ICR mice (20-30 g) were procured from the Shanghai Medical College, China. All the animals were housed in the laboratory for $12 \mathrm{~h}$ light/dark cycle, at a temperature of $25 \pm 2{ }^{\circ} \mathrm{C}$ and humidity of $60 \pm 5 \%$. All the animals were kept for a period of 7 days for the acclimatization to laboratory conditions with free access to normal standard chow diet and tap water. All the protocols of animal use were approved by the Institutional Animal Care and Use Committee (IACUC) of the Capital Medical University, China (IACUC/IAEC/2017/12).

\section{Induction of traumatic brain injury}

Marmarou's weight-drop model was used to induce TBI. All the animals were anesthetized and kept under the weight drop device on the platform. An incision of $1.5 \mathrm{~cm}$ on the midline of the scalp was made, which exposed the skull by removing fascia. On the skull, the weight of $200 \mathrm{~g}$ was released after locating the impact area (left anterior frontal area). Later, the suture was used to close the wound.

\section{Experimental procedure}

All the animals were kept for acclimatization for a period of seven days and separated into three groups such as the control group i.e. sham-operated group without weight drop and saline solution was administered; the negative control group i.e. TBI and admin- istered saline solution; vorinostat group that received $100 \mathrm{mg} / \mathrm{kg}$ of vorinostat intraperitoneally $30 \mathrm{~min}$ before the TBI induction. All the animals were anesthetized one day after the TBI induction and saline (0.9\%) followed by paraformaldehyde (4\%) was used for intracardial perfusion.

\section{Neurological score and brain water content}

All the animals were kept on a 45-cm-long metal wire between the two poles and animals were allowed for one min to traverse the wire. Estimation of latency was done within one min period of traverse from the wire and the score was given as per the gripping of the wire such as 0: unable to remain on the wire for < 30 s; 1: Failed to hold the wire with both hind and fore paws; 2: Hold the wire with hind and fore paws but not the tail; 3: Used its tail along with both fore paws and both hind paws; 4: Moved along the wire on all four paws and tail; 5: Score four point for ambulating down one of the posts used to support the wire. Brain water content was determined by removing the cerebellum and brain stem and weighing the remaining ipsilateral tissues immediately for the wet weight. Later, the same tissues were dried for 3 days at $80^{\circ} \mathrm{C}$ for the determination of dry weight. The water content was determined by the following formula. \%WC $=(\mathrm{WW}-\mathrm{DW}) / \mathrm{WW} \times 100$.

\section{Determination of oxidative stress parameters}

The phosphate buffer was used to homogenate the tissue sample by centrifuging it for $15 \mathrm{~min}$ at 12 000x. The level of malondialdehyde (MDA) and activity of glutathione peroxidase (GPx) and superoxide dismutase (SOD) was determined in the tissue homogenate as per the manufacturer's instructions using a spectrophotometer.

\section{Immunohistochemical staining}

Isolated brain tissues were fixed for one day in the $4 \%$ formalin and after dehydration, tissues were embedded in molten paraffin wax. Microtome was used to cut the tissue section and further incubate at $4^{\circ} \mathrm{C}$ for a period of $24 \mathrm{~h}$ with Nrf2. The phosphate-buffered saline solution was used to wash the tissue section for $15 \mathrm{~min}$, thereafter horseradish peroxidase-conjugated IgG was used to incubate the section for $60 \mathrm{~min}$. Immunolabeling was visualized by diaminobenzidine and counter stain with hema- 
toxylin. A light microscope was used to observe the count of immunohistochemical-positive neurons.

\section{Estimation of intracellular content of ATP}

A somatic cell assay kit was used to determine the level of ATP. Intracellular ATP was released by the lysis of viable mitochondria and luciferase enzyme. Orion L Microplate Luminometer was used for the luminescence analysis.

\section{Western blot assay}

Cells from the isolated brain were lysed in the buffer containing the protease inhibitor after washing it with PBS solution for the extraction of total proteins. Lysate was centrifuged for $10 \mathrm{~min}$ at $12000 \times$ at $4^{\circ} \mathrm{C}$. The BCA kit was used for the estimation of the concentration of total protein as per the manufacturer's instructions. Gel electrophoresis was used for the fractionation of protein and filter through the PVDF membrane. Later, primary antibodies such as anti-p-Akt, anti-Akt, anti-NF- $\kappa B$, anti-iNOS, anti-caspase and anti- $\beta$-actin were incubated with membrane at $4^{\circ} \mathrm{C}$ overnight. Further, the membrane incubated with an anti-mouse horseradish peroxidase-conjugated secondary antibody and chemiluminescence plus system was used for the estimation of band intensity.

\section{TUNEL Assay}

The brain was isolated from each animal and 4\% paraformaldehyde was used to fix the tissue. Brain tissues were embedded in paraffin wax and later sec- tioned into slices with 5-mm thickness. Dead End TM fluorometric TUNEL system kit was used to perform the TUNEL fluorescent assay as per the manufacturer's instructions. Slides were prevented from getting exposed under the direct sun light and cellular nuclei was stained using Hoechst staining. NIS-Elements BR image processing and analysis software was used for the estimating of TUNEL positive cells in the hippocampal region.

\section{RT-PCR}

RNA iso Plus was used for the extraction of total RNA from the ipsilateral cortex. Agarose gel electrophoresis (1\%) and spectrophotometer was used to determine the purity and concentration of total RNA. The Prime Script RT reagent kit was used to prevent the degradation of RNA by reverse-transcribing it into cDNA. The primers were designed according to PubMed GenBank and synthesized by Invitrogen Life Technologies (Shanghai, China). Real-time SYBR Green PCR technology was used for the analysis of RT-PCR.

\section{Results}

\section{Effect of vorinostat on the neurological score and brain water content}

The effect of vorinostat on the neurological score and brain water content in the TBI mice is shown in Figure 1. The neurological score was estimated by determining the grip test score scale. It was observed that the grip test score was significantly reduced $(p<0.01)$ and water content in the brain was significantly enhanced in the negative control
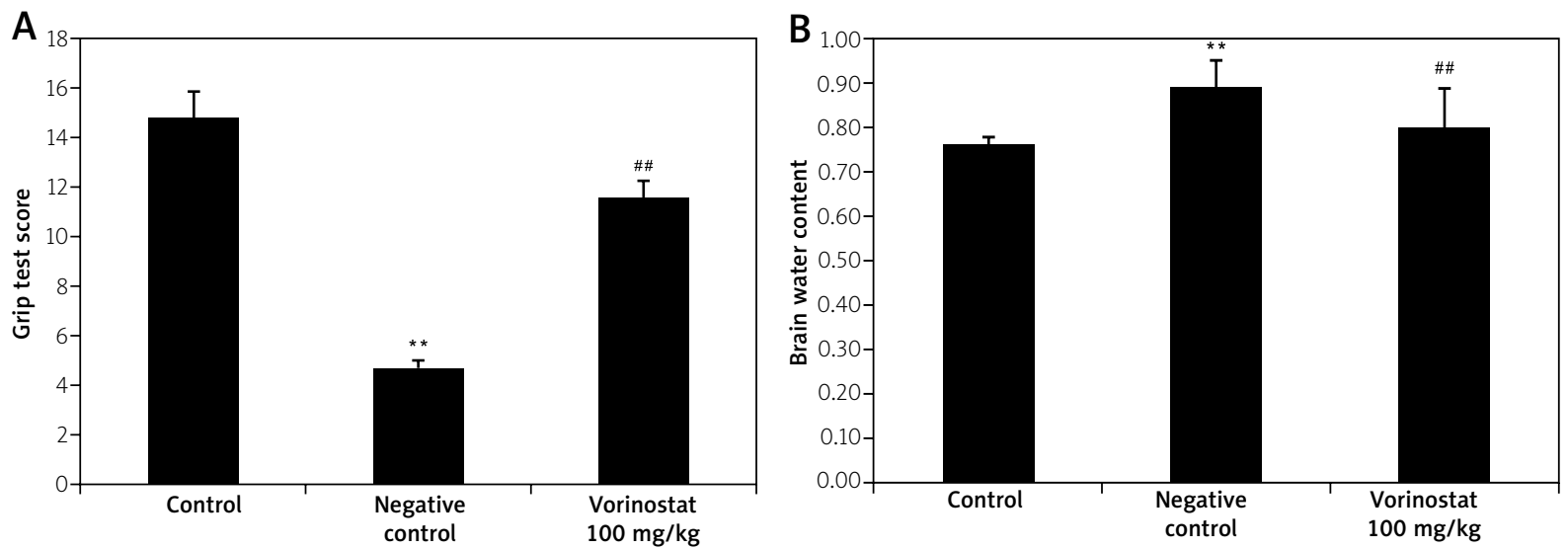

Fig. 1. Effect of vorinostat on the neurological score and brain water content in traumatic brain injury mice. Mean $\pm \operatorname{SEM}(n=10),{ }^{* *} p<0.01$ compared to the control group; ${ }^{\# \#} p<0.01$ compared to the negative control group. 
group compared to the control group of mice. However, treatment with vorinostat significantly reduced the altered level of grip test scores and water content in the brain of TBI mice.

\section{Effect of vorinostat on oxidative stress parameters}

The effect of vorinostat on oxidative stress parameters such as MDA, GPx and SOD in the tissue homog- enate of TBI mice is shown in Figure 2. It was observed that the level of MDA significantly enhanced $(p<0.01)$ and activity of GPx and SOD was significantly reduced in the tissue homogenate of the negative control group compared to the control group of mice. However, there was a significant decrease $(p<0.01)$ in the level of MDA and the activity of GPx and SOD was significantly enhanced in the vorinostat-treated group compared to the negative control group.
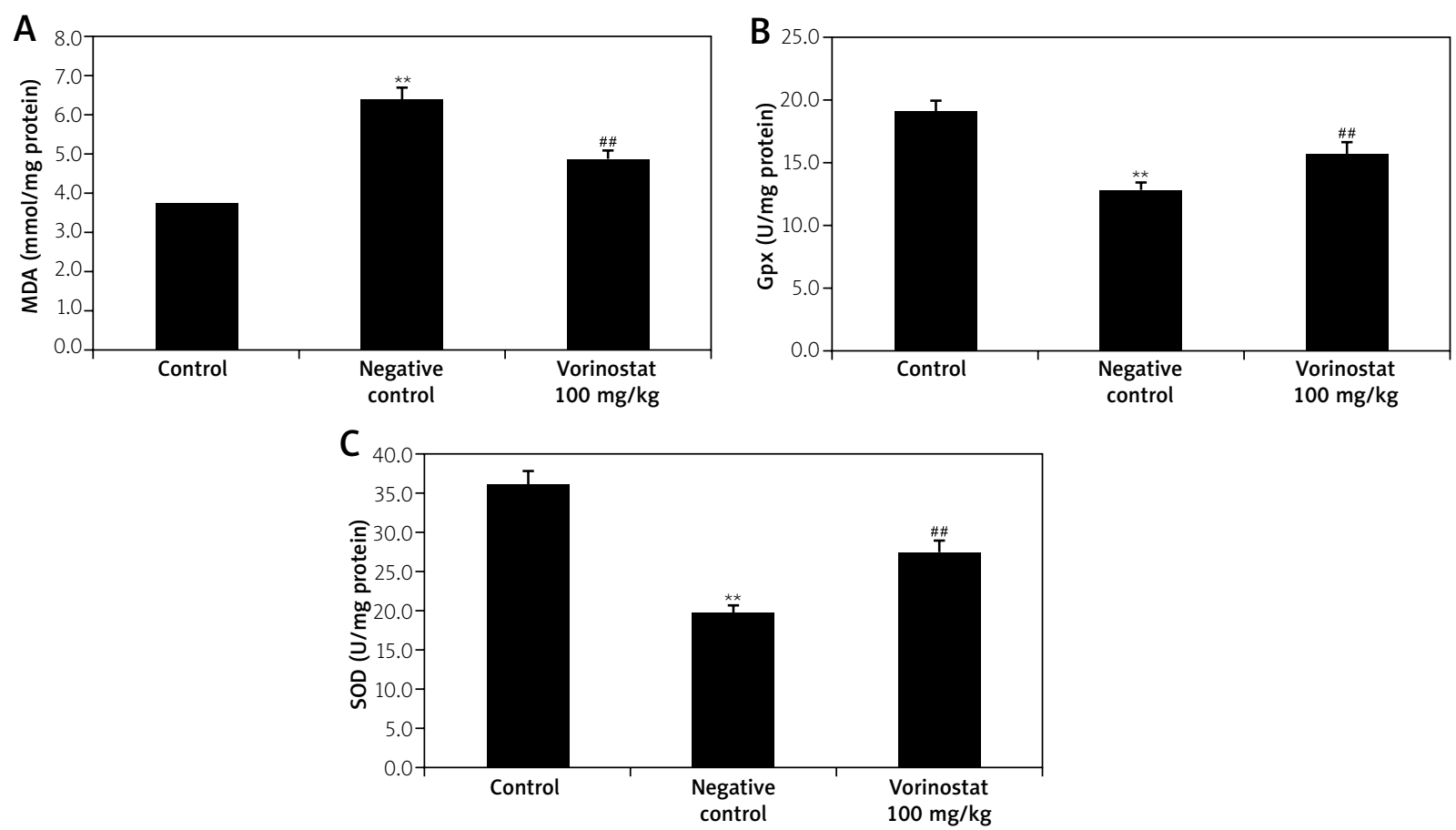

Fig. 2. Effect of vorinostat on oxidative stress parameters in the tissue homogenate of traumatic brain injury mice. Mean \pm SEM $(n=10),{ }^{* *} p<0.01$ compared to the control group; ${ }^{\# \#} p<0.01$ compared to the negative control group.
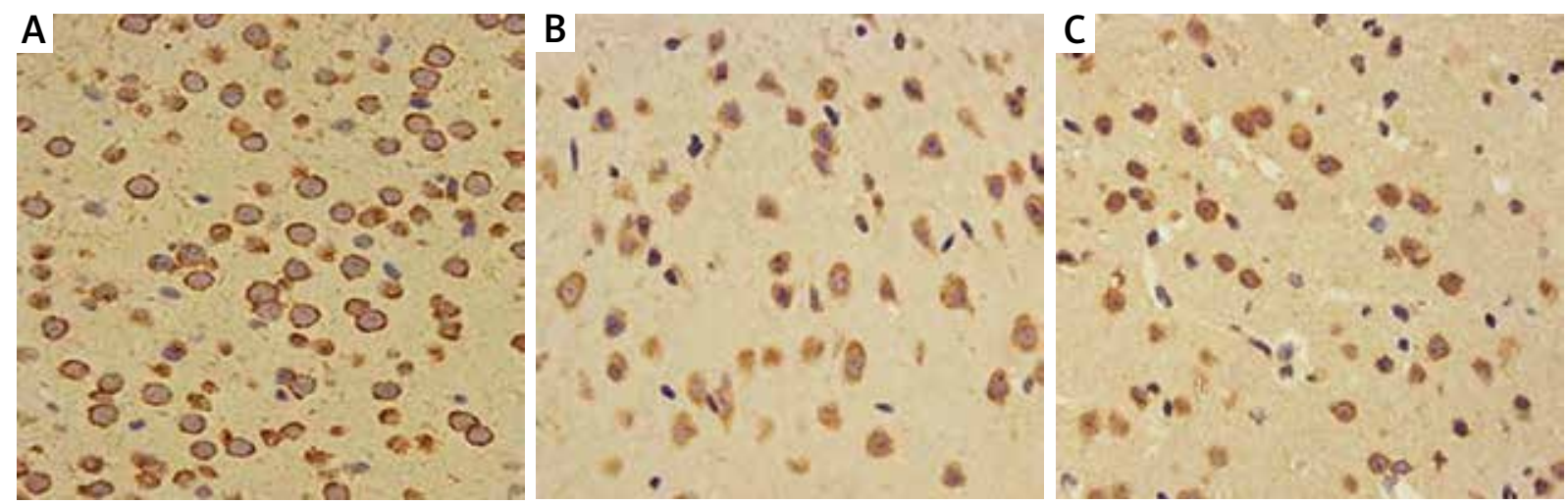

Fig. 3. Effect of vorinostat on Nrf2 in the neuronal tissue of traumatic brain injury mice by immunohistochemical staining. A) control, B) negative control, C) vorinostat $100 \mathrm{mg} / \mathrm{kg}$. 


\section{Effect of vorinostat on the concentration of Nrf2 in the nucleus}

The effect of vorinostat on Nrf2 in the neuronal tissue of TBI mice by immunohistochemical staining is shown in Figure 3. There was a significant increase in the number of Nrf2-immunoreactive neurons in the negative control group compared to the control group. However, the vorinostat-treated group shows a greater number of Nrf2-immunoreactivity detected neurons compared to the negative control group of mice.

\section{Effect of vorinostat on the ATP content}

The effect of vorinostat on intracellular ATP content in the neuronal tissue of TBI mice is shown in Figure 4. There was a significant decrease in the intracellular ATP content in the neuronal tissue of the negative control group compared to the control group. However, treatment with vorinostat significantly attenuates the intracellular ATP content in the neuronal tissue of TBI mice.

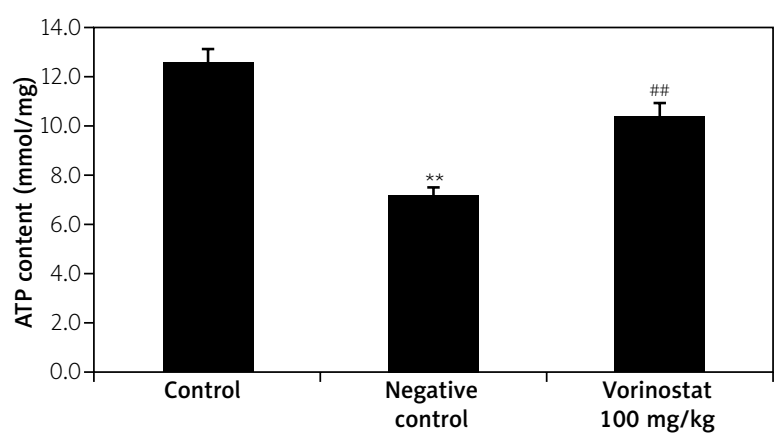

Fig. 4. Effect of vorinostat on intracellular ATP content in the neuronal tissue of traumatic brain injury mice. Mean \pm SEM $(n=10),{ }^{* *} p<0.01 \mathrm{com}-$ pared to the control group; ${ }^{\# \#} p<0.01$ compared to the negative control group.

\section{Effect of vorinostat on the expression of p-Akt, NF-kB, iNOS and caspase}

The effect of vorinostat on the expression of p-Akt, NF- $\kappa B$, iNOS and caspase in the neuronal tissue of TBI mice is shown in Figure 5. There was
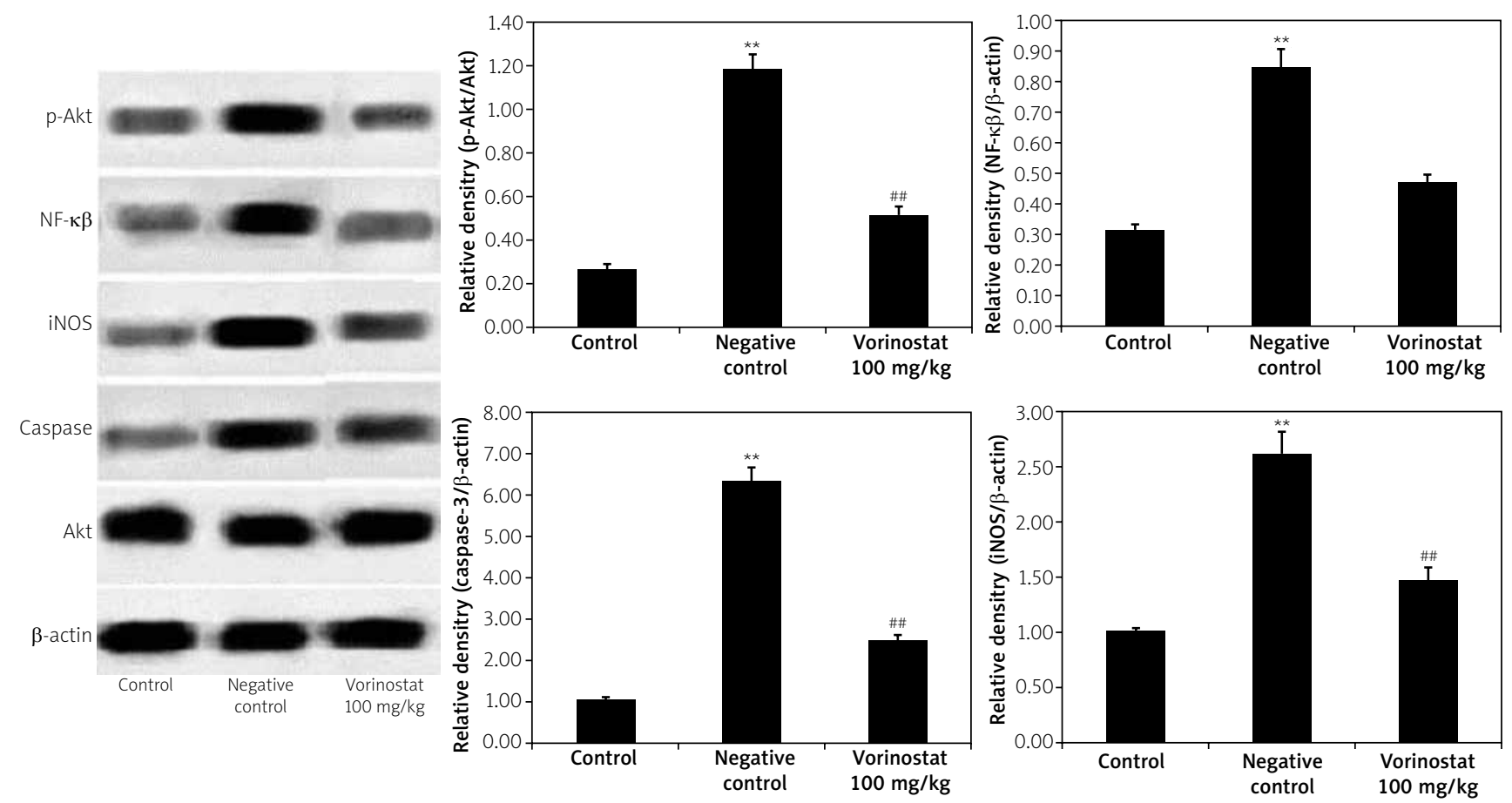

Fig. 5. Effect of vorinostat on the expression of p-Akt, NF- $\kappa B$, iNOS and caspase in the neuronal tissue of traumatic brain injury mice. Mean \pm SEM $(n=10),{ }^{\star *} p<0.01$ compared to the control group; ${ }^{\# \#} p<0.01$ compared to the negative control group. 


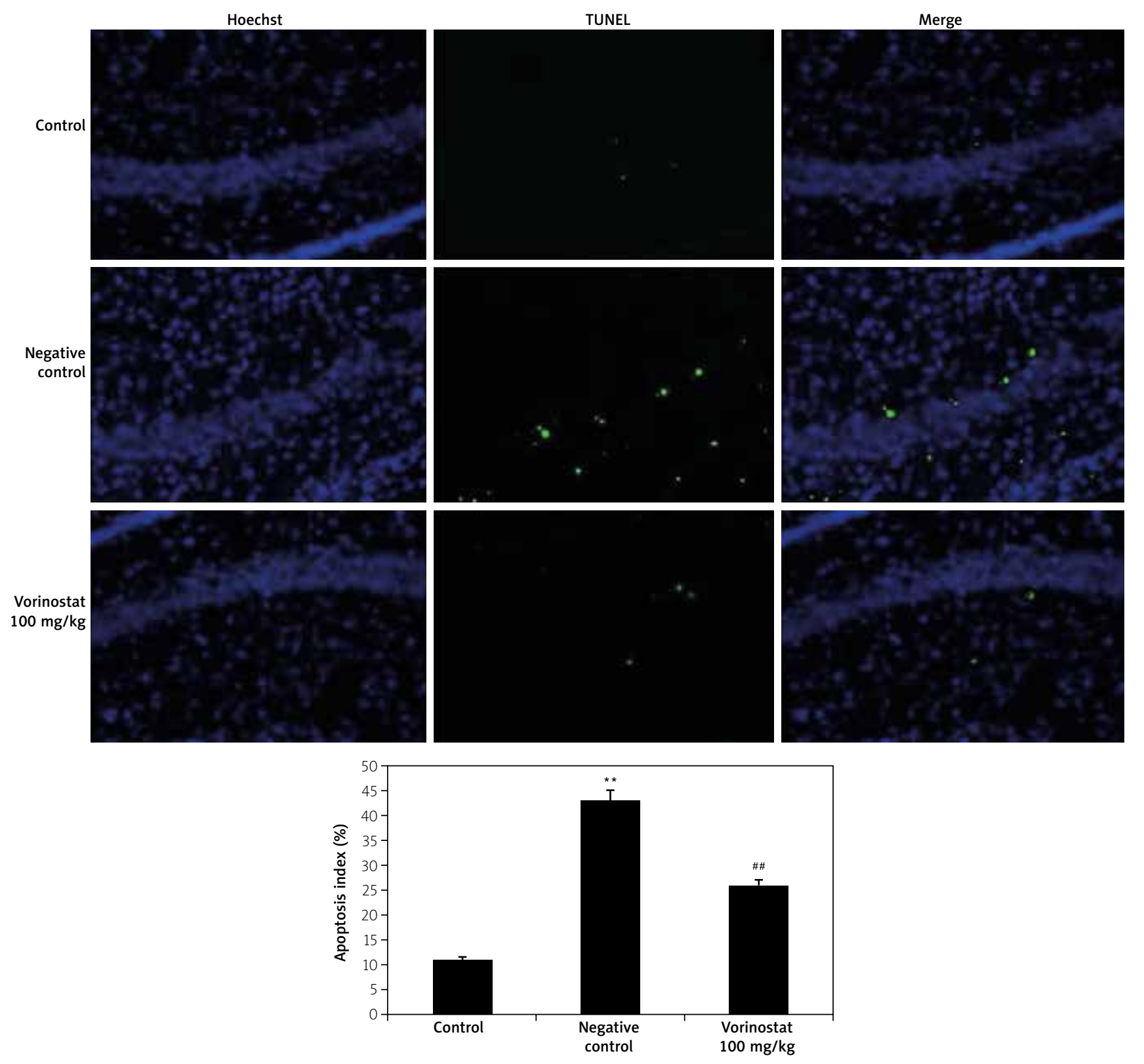

Fig. 6. Effect of vorinostat on the apoptosis index in the neuronal tissue of traumatic brain injury mice by TUNEL assay. Mean \pm SEM $(n=10),{ }^{* *} p<0.01$ compared to the control group; ${ }^{\# \#} p<0.01$ compared to the negative control group.

a significant increase $(p<0.01)$ in the $p-A k t, N F-\kappa B$, iNOS and caspase in the neuronal tissue of the negative control group compared to the control group of mice. However, treatment with vorinostat ameliorates the altered expression of $\mathrm{p}$-Akt, NF-kB, iNOS and caspase in the neuronal tissue of TBI mice.

\section{Effect of vorinostat on the apoptosis index}

The effect of vorinostat on the apoptosis index in the neuronal tissue of TBI mice by TUNEL assay is shown in Figure 6. It was observed that the number of TUNEL positive neuronal cells was significantly 

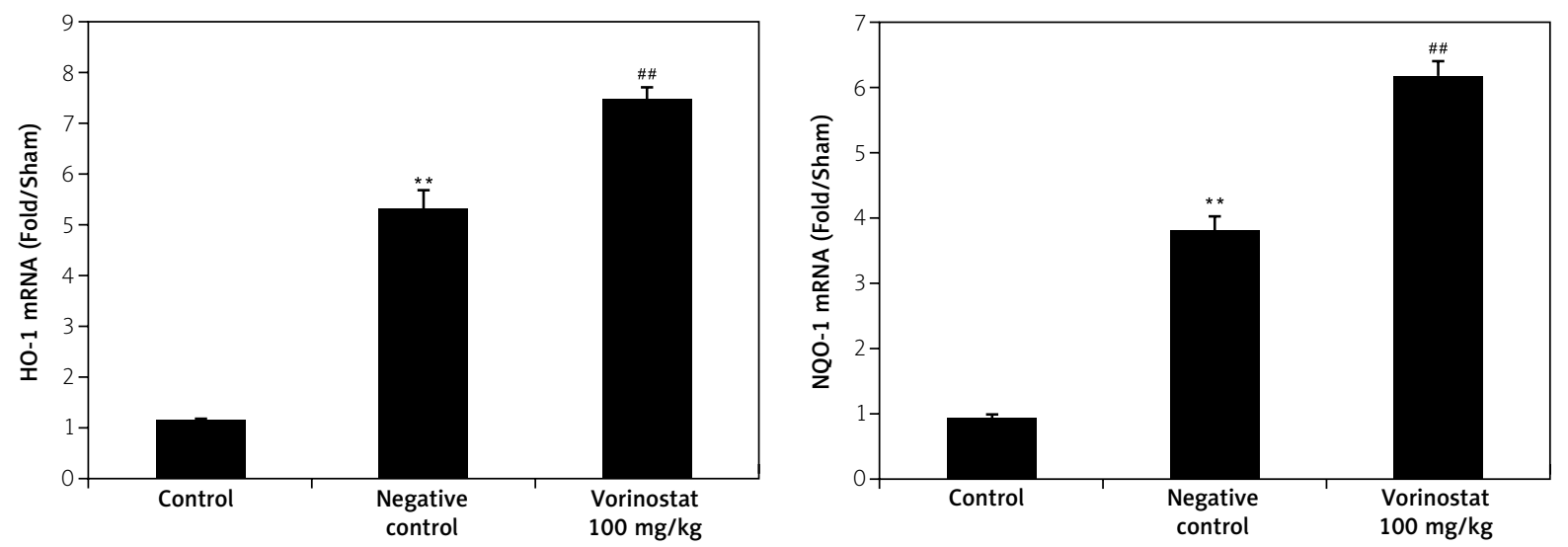

Fig. 7. Effect of vorinostat on the HO-1 and NQO-1 mRNA level in the neuronal tissue of traumatic brain injury mice. Mean \pm SEM $(n=10),{ }^{* *} p<0.01$ compared to the control group; ${ }^{\# \#} p<0.01$ compared to the negative control group.

enhanced $(p<0.01)$ in the negative control group compared to the control group of mice. However, the quantity of TUNEL positive cells was significantly reduced in the vorinostat group compared to the negative control group. Moreover, vorinostat also reduces the percentage of the apoptosis index significantly $(p<0.01)$ compared to the negative control group of mice.

\section{Effect of vorinostat on the HO-1 and NQO-1 mRNA}

The effect of vorinostat on the HO-1 and NQO-1 mRNA level in the neuronal tissue of TBI mice by using RT-PCR is shown in Figure 7. The mRNA level of HO-1 and NQO-1 was significantly enhanced in the tissue homogenate of the negative control group compared to the control group of mice. Moreover, treatment with vorinostat further enhances the mRNA level of HO-1 and NQO-1 compared to the negative control group of mice.

\section{Discussion}

In the modern society, TBI is a major health problem that causes disability and death in the young aged population. The present study evaluates the neuroprotective effect of vorinostat against TBI and also postulates the possible mechanism of its action. Marmarou's weight-drop model was used to induce $\mathrm{TBI}$ and the neurological score and brain water content was determined. Moreover, the effect of vori- nostat on the TBI was estimated by oxidative stress parameters, immunohistochemical staining, TUNEL assay, Western blot assay and RT-PCR.

According to the literature, inflammation, loss of ionic homeostasis, excitotoxicity, oxidative stress and altered vascular permeability contribute to the pathogenesis of the secondary injury in TBI [15]. Oxidative stress plays an important role in the pathology of TBI and alteration in the behavioral deficits [14]. In oxidative stress, production of free radicals enhanced that result in oxidation and nitration of proteins, lipid peroxidation and DNA damage [5]. In TBI, the level of MDA is reported to be enhanced and activity of GPX and SOD reduced in the neuronal tissues [17]. Data of the study reveal that treatment with vorinostat reduces the altered parameters of oxidative stress.

In several central nervous system (CNS) disorders, the antioxidant enzyme was induced due to Nrf2 and HO-1 and NQO1 like phase II enzyme was reported to be activated in TBI [18]. Thus, the Nrf2/ ARE pathway could be a therapeutic target for the development of a drug therapy for the management of the secondary injury in TBI [2]. Data of our study reveal that vorinostat reduces the altered level of Nrf2 in the nucleus of TBI mice. Moreover, the mRNA level of $\mathrm{HO}-1$ and $\mathrm{NQO} 1$ was reduced in the brain tissue of TBI mice.

The reported study reveals that in the secondary injury in TBI, neuronal apoptosis was induced due to oxidative stress and inflammatory response [4]. The 
results of the study show that vorinostat significantly reduces the $(p<0.01)$ apoptosis of neuronal cells compared to the negative control group.

\section{Conclusions}

The present study concludes that vorinostat attenuates the neuronal apoptosis in TBI mice by attenuating the altered iNOS/Nrf2/ARE pathway. Data of the study reveal that vorinostat has strong antioxidant activity and thereby protects the neuronal injury against TBI mice. Vorinostat could be used clinically for the management of neuronal injury.

\section{Acknowledgements}

All the authors of the manuscript are thankful to the Wuxi People's Hospital, China for providing the necessary facility for the presented work.

\section{Disclosure}

The authors declare no conflict of interest.

\section{References}

1. Bassett SA, Barnett MPG. The role of dietary histone deacetylases (HDACs) inhibitors in health and disease. Nutrients 2014; 6: 4273-4301.

2. Calkins MJ, Johnson DA, Townsend JA, Vargas MR, Dowell JA, Williamson TP, Kraft AD, Lee JM, Li J, Johnson JA. The Nrf2/ARE pathway as a potential therapeutic target in neurodegenerative disease. Antioxid Redox Signal 2009; 11: 497-508.

3. Chen X, Guo C, Kong J. Oxidative stress in neurodegenerative diseases. Neural Regen Res 2012; 7: 376-385.

4. Chen X, Wang H, Zhou M, Li X, Fang Z, Gao H, Li Y, Hu W. Valproic acid attenuates traumatic brain injury-induced inflammation in vivo: involvement of autophagy and the Nrf2/ARE signaling pathway. Front Mol Neurosci 2018; 11: 117.

5. Guo C, Sun L, Chen X, Zhang D. Oxidative stress, mitochondrial damage and neurodegenerative diseases. Neural Regen Res 2013; 8: 2003-2014.

6. Jeong Y-H, Park J-S, Kim D-H, Kim H-S. Lonchocarpine increases Nrf2/ARE-mediated antioxidant enzyme expression by modulating AMPK and MAPK signaling in brain astrocytes. Biomol Ther 2016; 24: 581-588.

7. Kinoshita K. Traumatic brain injury: pathophysiology for neurocritical care. J Intensive Care 2016; 4: 29.

8. Lohman RJ, Iyer A, Fairlie TJ, Cotterell A, Gupta P, Reid RC, Vesey DA, Sweet MJ, Fairlie DP, Differential anti-inflammatory activity of HDAC inhibitors in human macrophages and rat arthritis. J Pharmacol Exp Ther 2016; 356: 387-396.

9. Mann BS, Johnson JR, Cohen MH, Justice R, Pazdur R. FDA approval summary: vorinostat for treatment of advanced primary cutaneous T-cell lymphoma. Oncologist 2007; 12: 12471252.
10. McElvaney NG. Alpha-1 antitrypsin therapy in cystic fibrosis and the lung disease associated with alpha-1 antitrypsin deficiency. Ann Am Thorac Soc 2016; 13 Suppl 2: S191-196.

11. Nita M, Grzybowski A. The role of the reactive oxygen species and oxidative stress in the pathomechanism of the age-related ocular diseases and other pathologies of the anterior and posterior eye segments in adults. Oxid Med Cell Longev 2016; 2016: 3164734.

12. Pettke A, Hotfilder M, Clemens D, Klco-Brosius S, Schaefer C, Potratz J, Dirksen U. Suberanilohydroxamic acid (vorinostat) synergistically enhances the cytotoxicity of doxorubicin and cisplatin in osteosarcoma cell lines. Anticancer Drugs 2016; 27 : 1001-1010.

13. Popescu C, Anghelescu A, Daia C, Onose G. Actual data on epidemiological evolution and prevention endeavours regarding traumatic brain injury. J Med Life 2015; 8: 272-277.

14. Prins M, Greco T, Alexander D, Giza CC. The pathophysiology of traumatic brain injury at a glance. Dis Models Mech 2013; 6 : 1307-1315.

15. Quillinan N, Herson PS, Traystman RJ. Neuropathophysiology of brain injury. Anesthesiol Clin 2016; 34: 453-464.

16. Toklu HZ, Tümer N. Oxidative stress, brain edema, blood-brain barrier permeability, and autonomic dysfunction from traumatic brain injury. In: Kobeissy FH (ed.). Brain neurotrauma: Molecular, neuropsychological, and rehabilitation aspects. CRC Press/Taylor \& Francis, Boca Raton (FL) 2015.

17. Yang Y, Wang H, Li L, Li X, Wang Q, Ding H, Wang X, Ye Z, Wu L, Zhang $X$, Zhou M, Pan H. Sinomenine provides neuroprotection in model of traumatic brain injury via the Nrf2-ARE pathway. Front Neurosci 2016; 10: 580.

18. Zhang M, An C, Gao Y, Leak RK, Chen J, Zhang F. Emerging roles of Nrf2 and phase II antioxidant enzymes in neuroprotection. Prog Neurobiol 2013; 100: 30-47. 